\title{
Psychotherapy Research and the Study of the First Person Experience
}

\author{
Ps. Ricardo Pulido \\ Universidad Alberto Hurtado, Santiago, Chile
}

\begin{abstract}
Over the last years, the first person methodologies have been transformed into a valid and significant alternative, different from the classic quantitative and qualitative methods. In contrast with other perspectives, the first person approach considers that the subject is able to observe and give a direct account of his subjective process. However, this process, far from being spontaneous and automatic, requires a phenomenological training. The article discusses the notion of "the first person" starting with the works of Varela and Husserl's Phenomenology. We will review the principal procedures of this methodology and we will describe examples of this kind of research in the field of psychopathology, highlighting the contributions that this approach may provide in order to close the gap between practice and research.
\end{abstract}

Keywords: psychotherapy research, consciousness, phenomenology, the first person methodologies

\section{Introduction}

The problem of the first person and the third person views have been recently introduced as a main topic in the philosophy of mind and particularly in the study of consciousness. The distinction of these perspectives replaces, in some way, the classic mind/body problem, but not completely. In fact, in a very materialistic vision of the human being, as it prevails today, the mind has been usually understood in terms of brain activity, so for many years the problem has stopped being the mind or the mind body connection, and has become about the complexity of such a biochemical system. In other words, the question was no longer philosophical, but technical: a matter of time! However, in the last twenty years, thanks to the work of Francisco Varela, among others, it can be seen that some aspects of the human experience resists any third person or objective explanations, not because a provisional lack of technical knowledge, but because of their intrinsic subjective nature such as subjective experience, qualia or sensation and consciousness. In other words, the third person methodologies never could account for these kinds of phenomena and so a different approach becomes necessary to understand them. Of course there are experts, like Daniel Dennett, who thinks that these aspects of the human experience are mere epiphenomena that have no significant implications for life and human beings: a kind of anecdotal phenomena. As we will see, however, the first person view, although non-objective, constitutes the heart of our subjective life: We are born, we suffer and we die as the first person, as the very protagonist of our experience, and not as a biochemical machine.

The failure of Wundt's experimental project, based on an introspective methodology, was critical for the course of cognitive science development. For about 100 years, the first person data has been considered almost

Ps. Ricardo Pulido, Ph.D., Facultad de Psicología, Universidad Alberto Hurtado. 
unanimously as unreliable and dismissible information. In this ideological context, traditional psychotherapy research evolved, hardly looking for objectified diagnosis, outcomes, unspecific factors, process and treatments, "cleaning" the evidence of its subjective appearance, or in other words, making strong efforts to produce the third person data. In the last 50 years, we have seen wonderful progress in these areas: We have empirically validated many of our practices; we have recognized the crucial role of the therapeutic alliance; we have produced specifics, operatives and reliable diagnosis criteria, and so on. However, the gap between research and practice still remains: The scientific knowledge we have produced, has not been translated into better clinical practices.

There are many who think that time will close the gap that still we need to accumulate more third person knowledge. The vision of this paper, in contrast, is that we need to reintroduce the first person data in the field of psychotherapy research, exactly as it has been happening in cognitive science during the last 10-15 years. But this reintroduction needs an accurate reflection of what the first person data is and what it is not.

\section{Rediscovering the First Person Studies}

Chalmers (online), one of the most important experts in the field of consciousness studies, pointed out that "If it was not for the direct experience which all of us have of the first person, it would seem a ridiculous concept”. In fact, even if we cannot verify in an objective way, nobody could negate the very subjective sense to be conscious: Right now all of us feel ourselves as our own self and not as some kind of external evidence, like the blackboard and the chairs of this room. Please, consider for instance your body. It represents a sort of boundary phenomenon, in the sense that we can perceive it as an external object and as a lived experience; it depends on our attitude towards it. Now, consider the act of perceiving your body; it does not matter the way in which you feel it, the very act of feeling the body cannot be experiencing it as an external phenomena and nobody, except of you, can verify its existence and quality. Well, this is the first person view or perspective, and as we will see, it can be explored in a rigorous and useful manner.

To better understand the difference between the first person and the third person data, let me introduce a general distinction. We have three main sources of data or ways in which we can describe a phenomenon:

(1) The third person data: That includes all of the data that an external observer can collect in an objective, or pseudo-objective, manner: EEG (electroencephalogram) tracings, neuro-images, physiological measures like hart rate or skin conductivity, psychometrical measures like Intelligence coefficient, and even our well known valid and reliable self-report questionnaires such as symptom check lists, outcome questionnaires and the working alliance inventories;

(2) Opinions: Which are subjective forms to describe a phenomenon that could or could not personally imply the descriptor and could or could not be given by an expert, for example, clinical judgment, preference questionnaires, social representations, etc.;

(3) The first person data: Which are descriptions of a phenomenon that is personally implied by the person. They are not "objective", because by its mere nature, the phenomenon cannot be experienced by an external observer, and at the same time they are not mere "subjective opinions", because they need a rigorous observational training that implies the capacity to observe the phenomenon suspending judgment about them and taking a sort of reflexive distance from the experiences themselves.

There is a fourth source of data called the second person perspective, in which an expert deals with another's subjective experience. Rigorously, the second person data could be considered as an opinion 
produced by an expert, but because he helps others to produce the first person data, it is perhaps better to consider it as at mid-point between "opinions" and "the first person data" or as a variation of both.

Thus, to say the first person and the third person views, is not equivalent to say subjective and objective perspectives, since the subjective dimension may be described with natural or phenomenological attitudes and precisely this determines where a first person account could be considered as the first person data or as a subjective opinion. In such a sense, the first person view is different from other methods like introspection, free association or the first person account of past or actual experiences as the subject naturally experiences them. Let us see the main differences:

Introspection classically refers to the act of turning attention inward to accurately observe mental content, such a as thoughts and sensations, in order to determine regularities across subjects and establish the basic elements of the experience content. For example, an introspectionist researcher may be interested in the experience of "seeing red": How does it feel and which kind of sensations it produces? He will ask the experimental subject to see the red stimulus, to be aware of their sensations and then describe them choosing some pre-established categories like "relaxed or tense"; “expanded or contracted”, etc.;

In contrast, when we are interested in collecting the first person data, we will not be interested in the experience content such as private thoughts or psychological associations and emotions, but in the structure of the experience itself that we may describe beyond the specific and private subjective content. As pointed out by Bertossa and Ferrari (2005): We will look for "i soggettivi universali”. An example of this is the experience of the intuition evidenced by Petitmengin (1999): independently of which kind of particular intuition one may live, it is possible to describe precisely the different aspects and phases involved in the phenomenological experience, like for instance the moment just preceding the intuition "characterized by an empty passage... a moment of confusion, obscurity, silence” (p. 70). Moreover, when we collect the first person data, we must not give pre-established descriptive categories to the experimental subject, but just guide him to observe his own experience, suspending judgment about it and in a second moment help him to describe it in his own terms.

The first person data is not the set of the first person accounts of past experiences (i.e., symptoms, biographical history, and interpersonal events) or of individual's actual subjective state (i.e., emotions, expectancies and beliefs) that an expert analyzes to extract the underlying phenomenological experience. This is the classic qualitative research methodology in which we collect subjective accounts, through interviews or focus groups, to analyze them choosing different strategies and models (semiotic analysis, discourse analysis, grounded theory, etc.). Contrarily, the first person data requires that the subject directly observes his experience in order to make new and more specific distinctions that for many reasons were not available to his consciousness before.

The first person data is not free association. In free association the patient must refer, with no censure, all the contents that emerge in his mind. In this sense, it is similar to the suspending a judgmental attitude typical of the phenomenological inquiry (“don't think if it is good or bad and just refer it”), but differs from it because in free association the subject is not an active observer of his experience, but a passive narrator of his mental and affective process. The attention is not addressed towards the phenomenological landscape, but to private thoughts and emotions.

In summary, the first person data is not equivalent to introspection, free association or the first person account of past or actual experiences such as the subject naturally experiences them. As we can see, what characterizes the first person data is not the source of information (the subject of the gross-grain experience), 
but the phenomenological description of particular aspects of the experience itself that normally remain unconscious to the subject, and the methods and strategies used to obtain it (trained subjects and/or mediators).

An important assumption behind this concept of the first person data is that we have thoughts and pre-thought knowledge and we may access phenomenologically both of them. The major problem with subjective experience, as pointed out by Le Van Quyen and Petitmengin (2002), is that "a large part of our experience is pre-thought or unconscious", so a rich description requires that the person "must become conscious of his pre-thought knowledge” (p. 275). This is not a simple task, however, because above all we generally get confused between experience and representation, that is, many times we tend to experience what we think about a phenomenon and not what actually is taking place. Our beliefs about ourselves and the world deeply determine and structure the way in which we experience it. As Husserl's Phenomenology has evidenced, the natural way in which we experience the world, on one hand, tends to substitute many aspects of our actual phenomenological experience with past representations and ideas of similar experiences (deforming effect) and on the other hand, to discard from the field of consciousness the dimensions of the experience that disconfirm the representations we have made about the world (concealing effect). In other words: "We only perceive what we understand" (Petitmengin, 2006, p. 235).

The historical problems correlated with subjective experience's description would be more related to our phenomenological analphabetism-that is, we completely ignore our ability to be deeply aware of our subjective world - than to the instability and unreliability of the first person data by itself. In this sense, commonly men or women may be situated in the mid-point of a continuum of which extremes are a baby who is totally unable to communicate verbally his internal states and an expert in the description of his own experience. Thus, the first person data is not faulty, but it needs trained subjects and a trained mediator or inestigator.

Varela has been probably the first who pointed out the need to reintroduce the subjective experience in the field of consciousness studies using trained subjects. Together with Husserl, Varela (1996) believed that it was possible to establish a real science of the experience. Because knowledge, including scientific knowledge, rises up from our lived experience, he thought that we need a science of the experience, which would offer the fundamental base to the natural science that in turn gives sense and meaning to our scientific practice and knowledge. Of course, as in every science, it is necessary to develop specific and rigorous methods and Varela suggested the phenomenological reduction or "epoché" husserliana as the better way to explore and examine the experience itself. Briefly, this method consists of four basic steps:

(1) Phenomenological attitude: An internal disposition to suspend every belief and judgment about what is under examination. This does not mean that we have to suspend our thoughts, but that we can change the direction of our thought's movement, from the content to the thought's development;

(2) Intimacy and intuition: Maintaining the phenomenological attitude, we enhance intimacy with our experience, the field of the experience progressively appears less nebulous and more vivid, and it becomes increasingly evident. At the end of this process a new understanding of the phenomenon, a sort of intuition or insight, suddenly emerges: Eureka!

(3) Description and invariants: After intuition, we try to put into words or some equivalent symbolic system (graphic models, etc.) the significant aspect of the lived experience. In this way the observations become public and suitable intersubjective validation;

(4) Training and stability: An episodic incursion into consciousness is quite different from a systematic 
phenomenological reduction practice (Varela, 1996). It is not easy to maintain the phenomenological attitude, to let intuition emerge and to precisely describe the experience. That's why only with regular training and practice, such as in other disciplines and sciences, significant and reliable knowledge can be produced.

Using this method, we can collect rigorous observation of many aspect of the experience such as intentionality, attention, time consciousness, corporal representations, emotions, and so on. Furthermore, we can contrast this data with the third person observation, like neuro-images or EEG. Varela's (1996) research program, more well-known as neurophenomenology, consists of this: a way in which the first person and the third person perspectives interact in a synergetic and complementary dialogue, helping each other to produce better and more meaningful distinctions of our subjective experience and its biological correlations. A Tibetan monk, an expert in his subjective experience, sitting in the lab with electrodes in his head is a suggestive picture of this approach.

\section{Exploring and Understanding Psychopathological Dynamics From the First Person Perspective}

There are excellent examples of these kinds of studies. Let me introduce one that is particularly interesting for its therapeutic implications. Le Van Quyen and Petitmengin (2002) have recently conducted a study with epileptic patients using the first person and the third person data in a very complementary way. They start with the knowledge that, in contrast with historical representation of the disease ${ }^{1}$, "Epileptic seizures do not arise suddenly simply as the effect of random fluctuations of brain activities, but requires a process of pre-seizure changes that start long before” (p. 169). This process has been described both from a third person (pre-seizure state in the neuronal dynamics) and a first person view (mental state of the patient that includes warning symptoms or prodomes before his seizures). However, these first person descriptions were unspecific, since almost all of them derived from self-questionnaires, unable to account for a detailed description of pre-seizure experience. Moreover, for an epileptic patient, it is particularly difficult to describe his pre-seizure experience, not only because many times they occur while asleep, but also because he can present post-seizure amnesia, or his interior sensibility could be reduced by the effect of the antiepileptic drugs or because the prior signs of the seizure often cause a panicked reaction that obstructs his ability to be aware of the warning symptoms.

In this context, the authors proposed to use a first person methodology in order to collect rigorous and richer descriptions of the state of mind of epileptic patients before their seizures and verify where the preictal neuro-dynamic is or not lived by the patient in his subjective field. As we can imagine, however, introducing a patient to the phenomenological reduction, was a very hard task, so they used a second person method, which is a mid-point between expert opinions and the first person data. In this strategy, a mediator, expert in conducting phenomenological interviews, helps the patient to deeply feel his experience, observe it with no judgment and produce an accurate description of what he has lived. As we can see, there are many similarities with the psychotherapeutic dialogue, but the main difference is that the mediator is not a therapist, and does not help the patient, but the patient is there to "help" the mediator by sharing his experiences. The intentionality is not the same, and this configures a completely different intersubjective scenario, and consequently, other aspects of the subjective experience can be focused, observed, and described. Another main difference is that the interviewer, in contrast with psychotherapists, must be an expert of his own subjective experience in order to guide others to

\footnotetext{
${ }^{1}$ We can remember "The Karamasov Brothers" of Dostoyevsky, in which the impossibility to anticipate an epileptic seizure was a major medical argument in favor of Smerdjakov, the main suspect of Karamasov's father's murder.
} 
describe their own, so he must have a deep training in phenomenological reduction practices.

Therefore, in order to help the patients to become aware and express their pre-seizure experience, the authors used a specific interview. Briefly, the interview includes the following three phases ${ }^{2}$ (Petitmengin, 2005; 2006):

(1) Guiding the patient toward the concrete evocation of a particular preictal experience from the past... until he feels that he is "reliving" it...;

(2) Helping the patient to slow down the "film" of his experience, in order to become aware of his internal process and aspects of his experience which until then were pre-thought...;

(3) Enabling him to put into words his experience, with the help of a particular form of questioning. (op. cit., p. 176)

Once the first person descriptions of many patients have been collected, the authors have analyzed the recurrent accounts, comparing them and establishing a generic model of the dynamic structure of the experience, which includes for instance, dimensions such as anticipative sensations and protection activities. This representation of the subjective pre-seizure experience was then compared and correlated with the neuro-dynamics patterns of the phenomenon. The results were both interesting and promising: Several structures of the subjective experience had a specific counterpart in brain dynamics. This offered an extraordinary argument for the development of therapeutic strategies based on cognitive self-control. In fact, the authors suggest that patients could be trained to be increasingly conscious to their preictal state and consequently to develop opportune strategies to anticipate, stop or avoid the seizures.

Another good example of the first person methodology in the field of clinical studies is the work of Parnas, Saas and Zehavi from the Center for Subjectivity Research in Copenhagen. Very close to the German phenomenological psychiatric tradition, they developed an interesting understanding of schizophrenia starting from Husserl phenomenology. They argue that an accurate exploration of the phenomenological psychopathology of schizophrenic patients not only shows a detailed description of the syndrome as lived by the subject, but can also have explanatory relevance. They have pointed out that "Schizophrenia is best understood as a disorder of consciousness and self-experience (disturbed ipseity) that involves two key aspects: hyper reflexivity (forms of exaggerated and alienating self-consciousness) and diminished self-affection (a diminishing sense of existing as a subject of awareness or agent of action)” (Saas \& Parnas, 2007, p. 63). The authors suggest that these two subjective aspects would be at the bases of all the schizophrenic spectrum psychopathology, including negative symptoms and prodomes manifestations. An example of this can be the "gedankenlautwerden", a typical symptom of the schizophrenic phenomenology that is the experience of perceived thoughts or inner speech with acoustic or auditory qualities. In fact, it seems that what is obvious for ordinary people, the implicit sense of selfness linked to every act of awareness, is by no means evident in the schizophrenic experience. In other words, in a normal state of consciousness, the individual is aware of the noematic aspects of the experience and, at the same time, has an implicit sense of being the one who is experiencing this or that; but in the schizophrenic phenomenology, the content of the experience or the noematic, seems colonized by the noetic aspect or the intentional stream of awareness, which is constantly present, impeding the natural and "naïve" experience of the external world. When this happens, the internal world acquires concrete and perceptual qualities, so thoughts, will, emotions, the body and so on, are experienced as external things, such as the noematic, like clouds in the sky, as independent objects which do

${ }^{2}$ For a full description of the interview see Petitmengin 2005 and 2006. 
not have the flavor of "myness", like "my body", "my thought”, or "my will”.

From this understanding of the schizophrenic phenomenology, the authors developed the EASE (examination of anomalous self-experience) (Parnas et al., 2005) in order to explore the first person experience of the sense of self or ipseity in the first episode schizophrenic patients. They interviewed hundred consecutive patients in a period of four years, using a phenomenological interview approach, and they found more than fifty self-anomalous experiences common to many of the patients, that were clustered in the following five dimensions:

(1) Cognition and stream of consciousness: "a normal sense of consciousness as continuous over time, flowing, inhabited by one subject and introspectively transparent (immediately or directly given) in a nonspatial way” (p. 240). Includes experiences like: loss of thought ipseity, spatialization of experience and discontinuous awareness of own action;

(2) Self awareness and presence: "a normal sense of being (existence) involves automatic unreflected self-presence and immersion in the world (natural, automatic, self evident)” (p. 244). Includes experiences like: diminished sense of basic self, distorted first person perspective and hyper reflectivity;

(3) Bodily experience: “a normal sense of psychophysical unity and coherence, a normal interplay or oscillation of the body as 'lived from within' as a subject or soul (nonspatial, spiritual 'Leib') and of the body as an object (spatial and physical 'Körper')” (p. 252). Includes experiences like: mirror-related phenomena, somatic depersonalization and bodily disintegration;

(4) Demarcation/transitivism: "loss or permeability of self-world boundary” (p. 254). Includes experiences like: confusion with others, other transitivistic phenomena and passivity mood;

(5) Existential reorientation: "the patient experiences a fundamental reorientation with respect of his general metaphysical worldview and/or hierarchy of values, projects and interest” (p. 255). Includes experiences like: primary self-reference phenomena, feeling of centrality, and solipsistic grandiosity.

An interesting aspect pointed out by the authors is that these experiences, usually were so strange to the patients that they never communicated them to anyone else, not only because they could be embarrassed to share them with others or may have had some cognitive difficulties to produce linguistic reports, but beyond all these, by the fact that the major part of these experiences had a prereflective quality, thus "They are not explicit in the focus of thematic attention but constitute more the overall background of awareness" (Parnas et al., 2005, p. 237).

Again, we can see the necessity of the first and the second person methodologies in order to really explore the subjective experience, since frequently the experience itself is not clear to the subject. In fact, to conceptualize the experience, especially prereflectives ones, is not an easy, spontaneous and automatic process; rather, it becomes progressively accessible to oneself, and eventually to the other, only if the subject makes a conscious effort to explore his own mind and reflect upon his own experience. Moreover, this process often requires the presence of a second person who helps the subject to make a sharable account, by asking him for more detailed and precise descriptions. Actually, the presence of other person authentically interested in the subject experience and who is able to create a safe, non-judgmental, interpersonal environment, encouraging him to produce an intersubjective report that makes the prereflective experience accessible not only to the other one, but also to his own self.

Using this phenomenological approach, Parnas and his colleagues sought to describe many self-experiences of schizophrenic patients that they had never discussed before, and that of course is not 
described in the psychopathological manuals, nor in the DSM IV. And these are not mere descriptions of subjective landscapes of the patients, but significant aspects of the illness that give us a better understanding of the schizophrenia and of the patients who suffer it.

\section{Conclusion}

The work of Petitmengin and Le Van Quyen, and the studies of Parnas and his colleagues, show us how the first person approach may be very useful to better understand some psychopathological dynamics and to develop specific therapeutic strategies. This kind of research is perfectly applicable with many mental disorders, such as obsessive compulsive disorder, depression, panic disorder, personality disorders and so on. As many mindfulness based treatments have shown us, with regular practice the patients are able to be more aware of their mental and interpersonal process. Furthermore, this capacity could be trained not only for therapeutic purposes, but for contemporary research aims as well. Also, the first person view may be helpful to explore some aspects of the therapeutic relationship, like emotional regulation, the negative interpersonal process, alliance ruptures, etc.. The first person data could even be an excellent counterpart of the third person outcome measures, in order to evaluate therapeutic change and treatment effectiveness. Of course, the question is not the first person vs. the third person approach, but the necessity to reintroduce the crucial role of the first person in the study of human subjectivity and particularly in the field of psychotherapy, psychiatry and clinical psychology. It is convinced that a real science of the experience is possible and is an extraordinary way to close the gap between our scientific knowledge about psychotherapy and our clinical practices.

\section{References}

Bertossa, F., \& Ferrari, R. (2005). Lo sguardo senza occhio. Milano: Albo Versorio.

Chalmers, D. (n. d.). The first-person and third- person views (part I). Retrieved from http://consc.net/notes/first-third.html

Le Van Quyen, M., \& Petitmengin, C. (2002). Neuronal dynamics and conscious experience: An example of reciprocal causation before epileptic seizures. Phenomenology and the Cognitive Sciences, 1(2), 169-180.

Parnas, J., Moeller, P., Kircher, T., Thalbitzer, J., Jansson, L., Handest, P., \& Zahavi, D. (2005). EASE-scale: Examination of anomalous self-experience. Psychopathology, 38(5), 236-258.

Petitmengin, C. (1999). The intuitive experience. Journal of Consciousness Studies, 6(2-3), 43-47.

Petitmengin, C. (2005). Un exemple de recherche neuro-phénoménologique: l'anticipation des crises d'épilepsie. Intellectica, 40, 63-89.

Petitmengin, C. (2006). Describing one's subjective experience in the second person, an interview method for the science of consciousness. Phenomenology and the Cognitive Sciences, 5, 229-269.

Sass, L., \& Parnas, J. (2007). Explaining schizophrenia: The relevance of phenomenology. In M. C. Chung, K. W. M. Fulford, G. Graham (Eds.), Reconceiving schizophrenia (pp. 63-95). Oxford: Oxford University Press.

Varela, F. J. (1996). Neurophenomenology: A methodological remedy to the hard problem. Journal of Consciousness Studies, 3(4), 330-350. 